\title{
Prevalencia y Factores Asociados a Enfermedades de Transmisión Sexual en Menores Explotados Sexualmente en Bogotá, Colombia
}

\section{Sexually-transmitted disease prevalence and the factors associated with it in sexually-exploited children in Bogota, Colombia}

Ángela M. Pinzón-Rondón ${ }^{1,2}$, Timothy W.W. Ross ${ }^{3}$, Juan C. Botero ${ }^{4}$ y Margarita M. Baquero-Umaña ${ }^{1}$

\footnotetext{
1 Facultad de Medicina, Universidad del Rosario. Bogotá, Colombia, ampinzon@urosario.edu.co, marguis_2000@hotmail.com

2 Facultad de Salud Pública, University of Maryland. College Park, MD, USA, ampinzon@umd.edu

3 Fundación Social Fénix. Bogotá Colombia. fund_fenix@yahoo.com, timothy@etb.net.co

4 Facultad de Derecho, Georgetown University, USA. jcb89@law.georgetown.edu
}

Recibido 5 Septiembre 2008/Enviado para Modificación 5 Abril 2009/Aceptado 4 de Mayo 2009

\section{RESUMEN}

Objetivos Presentar características de un grupo de niños que han sido explotados sexualmente en Bogotá, determinar la prevalencia de Enfermedades de Transmisión Sexual (ETS) en estos menores y establecer factores asociados a estas enfermedades en este grupo.

Metodología Estudio descriptivo de corte transversal. Se revisaron historias clínicas de pacientes entre 10 y 17 años que asistieron a la consulta médica ambulatoria de la Fundación Renacer entre Enero del 2002 y Junio del 2004. El análisis incluyó estadísticas descriptivas, correlaciones y regresión logística binomial.

Resultados Se incluyeron 255 historias de menores. Características: 58,8 \% mujeres, 15,4 años edad promedio, 5,9 \% tenían alguna afiliación a seguridad social, 89,8 \% consumían alguna sustancia psicoactiva, $41,2 \%$ utilizaba algún método anticonceptivo, el 3,9 \% refirió utilizar condón en todas sus relaciones y 0,86 embarazos en promedio por adolescente femenina. La prevalencia de diagnósticos de enfermedades de transmisión sexual fue de 31,0 \%. Las enfermedades mas comunes fueron condilomatosis e infección por Neisseria gonorrhoeae. Las ETS se asociaron con número de sustancias psicoactivas consumidas y con uso poco frecuente de condón.

Conclusión La explotación sexual infantil es una realidad en Colombia. Este estudio confirma que las enfermedades de transmisión sexual son un problema importante en los menores explotados sexualmente en Bogotá. La prevención de explotación sexual infantil y la atención integral a estos menores, incluyendo la atención en salud, deben ser una prioridad. 
Palabras Clave: Abuso sexual infantil, enfermedades sexualmente transmisibles, Colombia, delitos sexuales (fuente: DeCS, BIREME).

\section{ABSTRACT}

Objective Presenting the characteristics of a group of children who have been sexually exploited in Bogota, determining the prevalence of sexually-transmitted disease (STD) in this group of children and establishing the factors associated with STD in this population.

Methodology This was a descriptive, cross-sectional study. The medical charts of children aged 10 to 17 years who sought medical attention at Fundación Renacer between January 2002 and June 2004 were reviewed. The analysis included descriptive statistics, correlations and binomial logistic regression.

Results 255 children's charts were included, producing the following characteristics: being female $(58.8 \%)$, average age was $15.4,5.9 \%$ were affiliated to social security, $89.8 \%$ engaged in substance abuse, $41.2 \%$ used family planning, $3.9 \%$ used a condom during every intercourse and there were 0.86 average pregnancies per female adolescent. Sexually-transmitted disease prevalence was $30.9 \%$. The most common diseases were condilomatosis and Neisseria gonorrhoeae infection. Substance abuse and infrequent condom use were associated with a diagnosis of STD.

Conclusion Child sexual exploitation is a reality in Colombia. In spite of the limitations of this study, it can be concluded that STDs are an important problem in the sexuallyabused child population in Bogota. Preventing child sexual exploitation and integral attention for these children (including health service provision) must become a priority.

Key Words: Child abuse, sexually-transmitted disease, Colombia, sex offence (source: $\mathrm{MeSH}, \mathrm{NLM}$.

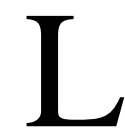

a explotación sexual infantil es un crimen que vulnera los derechos fundamentales de los niños. Es además una de las peores formas de maltrato infantil (1). A pesar de que existen convenios internacionales y legislación en todos los países para protegerlos, se estima que alrededor de 10 millones de niños(as) son explotados(as) sexualmente en el mundo y se sabe que este fenómeno está presente en prácticamente todas las culturas $(1,2)$.

En Colombia muchas veces se desconoce la explotación sexual infantil. Sin embargo, varias investigaciones científicas han documentado este fenómeno. Estas investigaciones se han centrado en prostitución y no arrojan una cifra unívoca. Las cifras disponibles van desde 4477 mujeres menores de 20 años ejerciendo la prostitución, hasta 35000 jóvenes vinculados a esta práctica en el país (3). En 1993 se encontraron 2959 menores de 18 años en prostitución solo en el centro de Bogotá (4). Factores como la vergüenza, el difícil acceso a la salud y la justicia, la falta de redes de apoyo social, y las amenazas y engaños por parte del explotador, hacen difícil el reporte de este fenómeno, lo cual lleva a pensar que las cifras reales 
son mucho más altas que las reportadas. Por otro lado, las organizaciones dedicadas a proteger a los niños tienden a presentar cifras más elevadas, posiblemente por un mayor conocimiento e interés en el tema (5). Lo que si se sabe es que la cantidad de niños y niñas que entran a la prostitución es cada día mayor y al parecer las edades de vinculación a la misma son cada vez más tempranas (4).

La Organización Internacional para las Migraciones ha determinado que Colombia es uno de los países de América Latina que más provee mujeres y niñas para tráfico de personas. Se ha detectado que Colombia envía mujeres y niñas a Europa, Asia, Estados Unidos y otros países latinoamericanos (6).

Las practicas de abuso y explotación sexual contra los menores en el país se ven reforzadas por: 1. La falta relativa de reconocimiento de los niños como seres en proceso de formación que lleva a los adultos a utilizarlos; 2 . El conflicto armado y el trafico de drogas, que no solo han propiciado la migración dentro del país especialmente de mujeres y niños -causando condiciones de miseria y contribuyendo a que menores desesperados sean fácilmente conducidos a actividades de explotación sexual (7)- sino que también han contribuido a la degradación de los valores éticos en la sociedad y al caos en los sistemas judiciales. Incluso se ha visto como algunos de estos menores son utilizados y explotados por integrantes de grupos armados (8), y; 3. La falsa creencia de que los menores no tienen posibilidad de portar enfermedades de transmisión sexual, que lleva a muchos adultos a preferirlos para satisfacer sus deseos sexuales (4).

La explotación sexual infantil, en si misma degradante, se asocia con abuso físico, psicológico y sexual, negligencia, abandono, carencia de atención médica, desescolarización, condiciones deficientes de vivienda y desplazamiento. Muchos menores explotados sexualmente tienen comportamientos de genitalidad compulsiva, consumo de sustancias psicoactivas, gestos e intentos de suicidio, violencia interpersonal, discriminación, embarazo precoz y múltiples enfermedades de transmisión sexual (9-12). Uno de los principales causantes de este problema es la migración $(12,13)$.

La prevalencia de enfermedades de transmisión sexual en menores colombianos explotados sexualmente es desconocida. La gran mayoría de estos menores no tienen servicios de salud e ignoran que pueden padecer estas enfermedades o lo niegan (4). Entre tanto, los adultos no buscan estos diagnósticos en los niños debido a creencias y actitudes negligentes hacia ellos (4). 
Un problema adicional que se presenta en este grupo de edad es que las infecciones de transmisión sexual (ITS) en los adolescentes repercuten con mayor impacto que en los adultos, debido a que se presentan cuando aún el individuo no ha completado su desarrollo. Las secuelas permanentes físicas y psicológicas suelen ser mayores y perdurar por un periodo más prolongado de tiempo (14-17).

Este artículo presenta: 1) una breve descripción de los niños victimas de explotación sexual que acudieron a la consulta médica del centro ambulatorio de la Fundación Renacer entre Enero del 2002 y Junio del 2004; 2) La prevalencia de diagnósticos de enfermedad de transmisión sexual y; 3) Los factores asociados a enfermedades de transmisión sexual en los mismos.

\section{METODOLOGÍA}

Estudio observacional, descriptivo, de corte transversal. El estudio se realizó revisando todas las historias clínicas de los pacientes entre 10 y 17 años que asistieron a la consulta ambulatoria de la Fundación Renacer-dedicada a la rehabilitación de menores explotados sexualmente en Bogotá- en el periodo comprendido entre Enero del 2002 y Junio del 2004. Se utilizó un formato para la extracción de los datos de las historias clínicas con instrucciones para obtener cada uno de los datos. Solo se tomó información de las citas a las que acudieron los menores durante el período del estudio. Se excluyeron únicamente dos historias clínicas porque tenían información faltante en más del $50 \%$ de las variables consideradas para el presente estudio.

Para describir la población de menores explotados sexualmente que acudieron a la consulta de la Fundación Renacer durante las fechas preestablecidas para el presente estudio se incluyen para todos los menores: la edad, el género, la afiliación a seguridad social, la afiliación al sistema educativo, el consumo de sustancias psicoactivas, el tipo de sustancias que consumen, la planificación familiar, el número de consultas a las que asistieron durante el período de estudio, el diagnóstico de enfermedades de transmisión sexual confirmadas y el tipo de las mismas. Para las menores de sexo femenino se incluyeron adicionalmente: el tipo de métodos que utilizan para planificar, el número de embarazos que han tenido, la presencia de embarazo en la actualidad, los partos y los abortos que reportan.

Las personas que recolectaron la información recibieron un entrenamiento acerca del mismo y contaron con los investigadores para resolver dudas durante el trabajo de campo. La información obtenida se validó en el $10 \%$ de los registros, en dos oportunidades. El protocolo contó con la aprobación del comité de ética en investigaciones de la Universidad del Rosario. 
El análisis descriptivo de los datos, fue seguido por correlaciones y finalmente por una regresión logística binomial jerárquica para determinar la influencia del condón y el consumo de sustancias psicoactivas en las ETSs.

\section{RESULTADOS}

Un total de 255 menores consultaron durante el período de tiempo establecido. De estos 255 menores atendidos solo 15 contaban con afiliación al Sistema de Seguridad social (5,9\%). El promedio de edad fue de 15,4 años, con un rango entre $10 \mathrm{y}$ 17 años. El 58,8 \% fueron de sexo femenino. El promedio de veces que asistieron a consulta médica 2,1. El 89,8 \% refirió consumir algún tipo de sustancia psicoactiva. El número de sustancias psicoactivas consumidas estuvo entre 0 y 7 con un promedio de 2,6. El 41,2\% de los menores utilizaban algún método de anticoncepción, la mayoría condón. El 57,6 \% de las menores reportaron haber estado o estar embarazadas, encontrándose un promedio de 0,86 embarazos con un rango de $0-4$. La Tabla 1 presenta información adicional.

Tabla 1. Información descriptiva de las variables

\begin{tabular}{|c|c|c|c|c|c|}
\hline Variables & $\mathrm{N}$ & Mín & Máx & Promedio & D.E.* \\
\hline Edad & 255 & 10 & 17 & 15,36 & 1,58 \\
\hline Número de consultas & 255 & 1 & 8 & 2,10 & 1,46 \\
\hline Número de Embarazos & 150 & 0 & 4 & 0,86 & 0,93 \\
\hline Número de Partos & 150 & 0 & 2 & 0,13 & 0,38 \\
\hline Número de Abortos & 150 & 0 & 2 & 0,45 & 0,62 \\
\hline \multirow[t]{2}{*}{ Número de sustancias que consume } & 255 & 0 & 7 & 2,59 & 1,73 \\
\hline & $\mathrm{N}$ & Frecuencia & $\%$ & & \\
\hline Género masculino & 255 & 150 & 58,8 & & \\
\hline Seguridad social & 255 & 15 & 5,9 & & \\
\hline Estudio & 255 & 26 & 10,2 & & \\
\hline Consumo de sustancias psicoactivas & 255 & 229 & 89,8 & & \\
\hline Consumo de alcohol & 255 & 138 & 54,1 & & \\
\hline Consumo de cigarrillo & 255 & 178 & 69,8 & & \\
\hline Consumo de marihuana & 255 & 127 & 49,8 & & \\
\hline Consumo de cocaína & 255 & 61 & 23,9 & & \\
\hline Consumo de bazuco & 255 & 35 & 13,7 & & \\
\hline Consumo de pegante & 255 & 90 & 35,3 & & \\
\hline Consumo de anfetaminas & 255 & 23 & 9,0 & & \\
\hline Consumo de benzodiacepinas & 255 & 6 & 2,3 & & \\
\hline Consumo de heroína & 255 & 3 & 1,2 & & \\
\hline Uso de algún Método anticonceptivo & 255 & 105 & 41,2 & & \\
\hline Anticonceptivos orales $(0=\mathrm{No})$ & 150 & 12 & 8,0 & & \\
\hline Óvulos $(0=$ No $)$ & 150 & 6 & 4,0 & & \\
\hline Dispositivo intra uterino $(0=\mathrm{No})$ & 150 & 12 & 8,0 & & \\
\hline Embarazo Actual $(0=\mathrm{No})$ & 150 & 41 & 27,3 & & \\
\hline ETS $(0=\mathrm{No})$ & 255 & 79 & 31,0 & & \\
\hline Uso de condón siempre & 255 & 10 & 3,9 & & \\
\hline Uso de condón casi siempre & 255 & 43 & 16,9 & & \\
\hline Uso de condón ocasional & 255 & 23 & 9,0 & & \\
\hline Uso de condón casi nunca & 255 & 179 & 70,2 & & \\
\hline
\end{tabular}


Se calculó una prevalencia de 31,0 \% de diagnósticos clínicos de enfermedades de transmisión sexual ya que se realizaron dichos diagnósticos a 79 menores. La Tabla 2 presenta un resumen de los diagnósticos encontrados. Algunos menores presentaron más de un diagnóstico.

Tabla 2. Diagnósticos de enfermedades de transmisión sexual

\begin{tabular}{llrr}
\hline & Diagnóstico & $\mathrm{N}$ & $\%$ \\
\hline Virales & VIH & 8 & 8,6 \\
& Hepatitis B & 1 & 1,1 \\
& Herpes Genital & 4 & 4,3 \\
& Condilomatosis Genital & 15 & 16,1 \\
& Condilomatosis Anal & 5 & 5,4 \\
& Linfogranuloma Venéreo & 1 & 1,1 \\
Bacterianas & Neisseria gonorhoeae & 19 & 20,4 \\
& C. Trachomatis & 10 & 10,8 \\
& Sífilis & 13 & 14,0 \\
& Enfermedad Pélvica Inflamatoria & 16 & 17,2 \\
Total (N) & Forúnculo Genital & 1 & 1,1 \\
& & 93 & 100,0 \\
\hline
\end{tabular}

Las correlaciones bivariadas se encuentran en la Tabla 3. El 95,8 \% de los adolescentes de sexo masculino consumían sustancias psicoactivas, mientras que el 77,8 \% de las adolescentes de sexo femenino lo hacían. Esta diferencia es estadísticamente significativa ( $\mathrm{p}<0,001)$. El consumo de cualquier tipo de sustancia psicoactiva es mayor en hombres excluyendo las benzodiacepinas para las cuales las mujeres presentan un mayor consumo. Los adolescentes que no se encontraban estudiando y aquellos no vinculados a seguridad social presentaron un mayor consumo de éstas. Los adolescentes de sexo masculino usaban el condón con más frecuencia que las adolescentes de sexo femenino ( $\mathrm{p}<0,01)$.

Tabla 3. Correlación de las variables en el modelo de regresión logística

\begin{tabular}{|c|c|c|c|c|c|c|c|c|c|c|}
\hline & 1 & 2 & 3 & 4 & 5 & 6 & 7 & 8 & & 10 \\
\hline 1 ETS & 1 & & & & & & & & & \\
\hline 2 No. sustancias que consume & $0,15^{*}$ & 1 & & & & & & & & \\
\hline 3 Uso de condón siempre & $-0,1$ & $0,16^{*}$ & 1 & & & & & & & \\
\hline 4 Uso de condón casi siempre & $-0,1^{*}$ & $-0,1$ & $-0,1$ & 1 & & & & & & \\
\hline 5 Uso de condón ocasional & 0,08 & $0,11 \dagger$ & $-0,1$ & $-0,14^{*}$ & 1 & & & & & \\
\hline 6 Uso de condón casi nunca & $0,11 \dagger$ & $-0,1$ & $-0,3^{\star \star \star}$ & $-0,69^{\star \star \star}$ & $-0,48^{\star \star *}$ & 1 & & & & \\
\hline 7 Edad & 0,05 & 0,04 & -0 & 0,05 & 0,08 & $-0,08$ & 1 & & & \\
\hline 8 Genero & $-0,1 \dagger$ & $-0,3^{* * *}$ & -0 & $-0,11 \dagger$ & $-0,15^{*}$ & $0,204^{* *}$ & 0,01 & 1 & & \\
\hline 9 Seguridad social & $-0,1$ & $-0,1 \dagger$ & 0,04 & $-0,07$ & 0,04 & 0,017 & 0,01 & 0,006 & 1 & \\
\hline 0 Estudio & 0,02 & $-0,3^{* * *}$ & -0 & 0,09 & $-0,06$ & $-0,04$ & $-0,09$ & $-0,01$ & 0,03 & 1 \\
\hline
\end{tabular}


La Tabla 4 presenta los modelos de regresión logística binomial para enfermedades de transmisión sexual en la población estudiada. A mayor número de sustancias psicoactivas consumidas por el menor mayor probabilidad de sufrir alguna enfermedad de transmisión sexual. Los adolescentes que utilizan el condón siempre o casi siempre tienen un menor riesgo de sufrir enfermedades de transmisión sexual cuando se comparan con aquellos que casi nunca utilizan el condón. Se puede apreciar que el incremento en una sustancia consumida aumenta el riesgo de sufrir alguna enfermedad de transmisión sexual en un 19,6\%.

Tabla 4. Modelos de regresión logística binomial para ocurrencia de ETS en menores explotados sexualmente

\begin{tabular}{|c|c|c|c|c|c|c|c|c|}
\hline & \multicolumn{4}{|c|}{ Modelo 1} & \multicolumn{4}{|c|}{ Modelo 2} \\
\hline & B & S.E. & Sig. & $\mathrm{RR}$ & $B$ & S.E. & Sig. & RR \\
\hline Constante & $-1,14$ & 0,27 & $* * \star$ & 0,32 & $-2,30$ & 1,48 & + & 0,10 \\
\hline Numero de sustancias que consume & 0,19 & 0,08 & * & 1,21 & 0,18 & 0,09 & * & 1,20 \\
\hline Uso de condón siempre & $-1,88$ & 1,08 & $\dagger$ & 0,15 & $-1,90$ & 1,08 & $\dagger$ & 0,15 \\
\hline Uso de condón casi siempre & $-0,98$ & 0,44 & * & 0,38 & $-1,19$ & 0,46 & $\star \star$ & 0,30 \\
\hline Uso de condón ocasional & 0,25 & 0,46 & & 1,28 & 0,12 & 0,48 & & 1,13 \\
\hline Uso de condón casi nunca & Omitido & & & & Omitido & & & \\
\hline Edad & & & & & 0,10 & 0,09 & & 1,10 \\
\hline Genero & & & & & $-0,48$ & 0,30 & & 0,62 \\
\hline Seguridad social & & & & & $-0,60$ & 0,68 & & 0,55 \\
\hline Estudio & & & & & 0,67 & 0,48 & & 1,96 \\
\hline R Square & 0,085 & & & & 0,116 & & & \\
\hline
\end{tabular}

$\dagger p<0.1,{ }^{*} p<0.05,{ }^{* *} p<0.01,{ }^{* * *} p<0.001$

\section{DISCUSIÓN}

Este estudio es el primero en presentar la prevalencia y factores asociados a las enfermedades de transmisión sexual en un grupo de adolescentes explotados sexualmente en Colombia. Los resultados muestran la existencia de la explotación sexual infantil en el país y de enfermedades de transmisión sexual secundarias a la misma.

Aunque son pocos los menores que se atreven a identificarse como explotados y a buscar atención médica (18), un total de 255 menores de edad explotados sexualmente acudieron a la consulta médica del centro ambulatorio de la Fundación Renacer entre enero del 2002 y junio del 2004. Las características demográficas de la población estudiada se corresponden con las reportadas por otras investigaciones en esta población en Colombia $(8,18)$. La asociación de la explotación sexual infantil y consumo de sustancias psicoactivas que ha sido ampliamente reportada en la literatura $(8,11,12,18)$ se confirma en este estudio al encontrar que el $89,8 \%$ de estos menores refieren consumo de alguna de estas sustancias. El consumo de pegante y cocaína no había sido reportado con anterioridad (8). 
La falta de afiliación al sistema de seguridad social es preocupante. Esto se debe en parte a que la mayoría de estos menores no vive con sus padres o con un adulto que tenga la patria potestad y pueda solicitar su afiliación. La experiencia de la Fundación Renacer ha demostrado que los menores presentan grandes dificultades en manejar los procedimientos burocráticos necesarios para conseguir su registro civil o solicitar la encuesta socio-económica para ingresar al sistema subsidiado de salud (SISBEN). Además, en general los menores no tienen confianza en las instituciones de protección porque han sido vulnerados por todas las personas en quienes han confiado y no quieren ser recluidos en contra de su voluntad u obligados a realizar algo que no quieren.

La prevalencia de enfermedades de transmisión sexual encontrada en la población estudiada fue de $31 \%$. Estudios en otros países han mostrado prevalencias de enfermedades de transmisión sexual en menores explotados sexualmente entre 21 y $71 \%$ dependiendo del grupo poblacional (19) y hasta del $38 \%$ exclusivamente para VIH (20,21). Es necesario aclarar que es muy posible que exista un subdiagnóstico de enfermedades de transmisión sexual en este estudio. Los menores no tuvieron la posibilidad de recibir un estudio completo, dado su antecedente de explotación sexual, para determinar la presencia de enfermedades de transmisión sexual asintomáticas o con síntomas indefinidos. Esto debido a su falta de afiliación a seguridad social, a la falta de recursos en la Fundación Renacer y en general a la ausencia de un servicio de salud especializado y asequible para ellos.

La condilomatosis, la infección por Neisseria gonorrhoeae, la enfermedad pelvica inflamatoria y la sífilis fueron las enfermedades diagnosticadas con mayor frecuencia. Estas estuvieron seguidas por Chlamydia trachomatis y VIH. Las enfermedades más comúnmente diagnosticadas -condilomatosis e infección por Neisseria gonorrhoeae- suelen producir síntomas y signos obvios que facilitan su diagnóstico. Esto podría explicar porque se encontraron más frecuentemente en el presente estudio.

Como ejemplo para dimensionar los resultados del presente estudio, al calcular la incidencia de VIH encontrada en esta población se obtiene una cifra de 3137 por 100000 habitantes. Esta cifra es extremadamente alta, sobre todo si se compara con la incidencia de VIH en Bogotá, que para el año 2004 fue de 10 por 100000 habitantes (22).

Esta investigación presenta evidencia que soporta la hipótesis de que las enfermedades de transmisión sexual en esta población están asociadas al consumo de sustancias psicoactivas y al uso inadecuado de condón. Esto incluso después de 
controlar por genero, edad, estudio y afiliación a seguridad social. La asociación positiva entre enfermedades de transmisión sexual y número de sustancias psicoactivas se explica porque el uso de sustancias psicoactivas es un factor de riesgo adicional para la ocurrencia de enfermedades de transmisión sexual, porque los menores bajo los efectos de dichas sustancias olvidan con mayor facilidad su protección personal (18), porque el abuso de sustancias incrementa la explotación sexual ya que los menores se ven obligados a incrementar esta práctica para poder financiar la compre de las mismas (18) y porque el uso de sustancias afecta el sistema inmune aumentando la susceptibilidad a infecciones $(23,24)$. En cuanto a la asociación de enfermedades de transmisión sexual y uso inadecuado de condón, esta simplemente confirma los efectos protectores del condón ante dichas enfermedades. Esta investigación encontró una asociación débil entre aquellos que usan el condón siempre y aquellos que casi nunca lo usan posiblemente porque solo un $3,9 \%$ de la muestra refirió usar el condón siempre. En la población general colombiana se ha reportado un uso de condón en todas las relaciones sexuales del $44 \%$ (25).

La tasa de embarazos y abortos en esta población es extremadamente alta. El $57,6 \%$ de las mujeres reportaron estar o haber estado embarazadas y esto contrasta con el $20 \%$ encontrado por Profamilia utilizando datos de mujeres en este mismo grupo de edad entrevistadas durante la Encuesta Nacional de Salud (25). Esto sumado a la prevalencia de enfermedades de transmisión sexual concuerda con la carencia de planificación familiar y la falta de protección adecuada en estos menores.

Este estudio tienemúltiples limitaciones dentro de las que se encuentran: 1. Presenta los resultados de un grupo limitado de niños y no de una muestra aleatoria de los menores explotados sexualmente en Bogotá, Colombia; 2. No permite determinar la prevalencia real de infecciones de transmisión sexual en la población estudiada por la forma en que fueron realizados los diagnósticos. La falta de ayudas diagnósticas impide conocer la magnitud real del fenómeno y; 3 . Las variables incluidas son escasas, teniendo en cuenta la complejidad de la problemática de las enfermedades de transmisión sexual en menores explotados sexualmente en Colombia.

A pesar de estas limitaciones este estudio permite comprobar: 1) la situación de vulnerabilidad en la que se encuentran los adolescentes explotados sexualmente en Colombia, 2) la urgente obligación de realizar prevención, tratamiento oportuno y rehabilitación a esta población, y 3) la necesidad de realizar procesos completos de investigación encaminados a solucionar el problema. 
En opinión de los autores no es adecuado considerar a la explotación sexual infantil como un trabajo, ya que en si misma es una de las peores formas de maltrato infantil. Sin embargo, si se la considera como tal, es sin duda una ocupación muy peligrosa (26) que se acompaña usualmente por consumo de sustancias psicoactivas, enfermedades, violencia, discriminación, actividades criminales y daños al desarrollo psicológico (12).

Colombia ha hecho un esfuerzo para mejorar la situación de estos niños. La instauración del nuevo código del menor es un ejemplo del mismo. Sin embargo hay mucho mas por hacer para evitar que este fenómeno se presente y para garantizar la recuperación de los adolescentes que ya son víctimas del mismo.

La prevención primaria, esto es evitar que los niños ingresen a la explotación sexual, es la única solución realmente adecuada. Para lograrlo algunos adolescentes colombianos en Bogotá y Pereira que han sido víctimas de la misma y que participaron en grupos focales durante el año 2005 aportaron las siguientes recomendaciones: 1) Los padres deben cuidar mejor a sus hijos, 2) a los menores se les debe creer cuando reportan este tipo de fenómenos, 3) los explotadores deben ser castigados, 4) se necesitan más programas para rehabilitar a las víctimas y 5) es necesario controlar el abuso de sustancias psicoactivas (2).

El uso de mecanismos para prevenir el riesgo ha sido propuesto para mejorar la salud de los trabajadores sexuales. Dentro de estos mecanismos se encuentran la educación por pares, el empoderamiento de la población, la atención especializada, la no criminalización, la educación a la población general para evitar estigmas, el uso de condón y el incremento de redes de protección en la comunidad $(12,13)$. Estos mecanismos sin embargo no son medidas completamente aceptables ya que asumen la presencia de los niños en estas actividades que deberían estar completamente prohibidas para ellos.

En conclusión, las enfermedades de transmisión sexual son un problema serio para los niños(as) y adolescentes explotados sexualmente. Son por lo tanto un problema de salud pública que requiere manejo, comenzando por un diagnóstico adecuado de las mismas. Éstas se acompañan por otros problemas como lo son la falta de seguridad social, el embarazo precoz, el consumo de sustancias psicoactivas, y el déficit en el uso de métodos de anticoncepción y de protección. El problema requiere un compromiso serio y claro de la sociedad que a criterio de los autores no se ha dado hasta la fecha en Colombia. 
Este trabajo se enfoca en uno de los múltiples efectos negativos que la explotación sexual trae a los niños -las enfermedades de transmisión sexual. Es importante recordar que este es solo uno entre tantos. La violencia sexual y el abuso psicológico al que estos menores están expuestos traen consecuencias muy negativas que generalmente los acompañan por el resto de la vida (27)

\section{REFERENCIAS}

1. Willis BM, Levy BS. Child prostitution: global health burden, research needs, and interventions. Lancet 2002 Apr 20; 359(9315):1417-22.

2. Ramirez C, Cuadros MI, García Cl, Orjuela L. Current Status of sexual abuse and exploitation of boys, girls and adolescents-the case of Colombia and Brazil. Final Technical Report. Bogota: Save the children UK programa suramérica; 2005 Apr. (Sponsored by Save the children UK).

3. Sánchez S. El caso de Colombia, violencia y explotación sexual en América Latina y el Caribe. Montevideo: Instituto Iberoamericano del niño OEA; 1999.

4. Fundación Renacer. La prostitución infantil en el centro de Bogotá. Bogotá: Cámara de Comercio de Bogotá; 1993.

5. Ennew J, Gopal K, Heeran J, Montgomery H. How can we measure the commercial sexual exploitation of children? Nueva York: UNICEF, University of Cambridge; 1996.

6. Organización Internacional para las Migraciones (OIM). Dimensiones de la trata de personas en Colombia. Bogotá: Panamericana formas e impresos SA; 2006.

7. Jeffery N, Carr-Lemke T. The Effects of Armed Conflict on Children in Colombia. Bogota: Save the Children; 2004.

8. Fundación Restrepo Barco, Plan Internacional, Fundación Renacer. Caracterización de las dinámicas de la ESCNNA en Arjona y Malagana (Bolívar), Soledad, Malambo y Sabanagrande (Atlántico), Quibdó (Chocó), Medellín (Antioquia), Sincelejo (Sucre), Montería (Córdoba) Cúcuta (N. de Sder.) y Cali (Valle). Colombia: Fundación Restrepo Barco, Plan Internacional, Fundación Renacer; 2004-2005.

9. Carvajal G. Adolecer: La aventura de una metamorfosis. Bogotá: Tiresias; 1994.

10. Dührssen A. Psicoterapia en niños y adolescentes. México: Fondo de cultura Económica; 1998.

11. Goti M. La comunidad terapéutica: un desafío a la droga. Buenos Aires: Nueva visión; 1990.

12. Rekart ML Sex-work harm reduction. Lancet. 2005 17;366(9503):2123-34.

13. Rushing R, Watts $C$, Rushing $S$. Living the reality of forced sex work: perspectives from young migrant women sex workers in northern Vietnam. J Midwifery Womens Health. 2005 Jul-Aug;50(4):41-4.

14. Burstein GR, Gaydos CA, Diener-West M, Howell MR, Zenilman JM, Quinn TC. Incident Chlamydia trachomatis infections among inner city adolescent females. JAMA. 1998 Aug 12;280(6):521-6.

15. Division of STD Prevention U.S. Department of Health and Human Services. Sexually transmitted disease surveillance. Atlanta: U.S. Department of Health and Human Services; 1998.

16. Friedman SB, Schonberg SK, Alderman EM, Fisher MM. (2nd edition) Comprehensive adolescent health care. St Louis: Mosby; 1998.

17. Des Jarlais DC, Hagan H, Friedman SR, Friedmann P, Goldberg D, Frischer M, et al. Maintaining low HIV seroprevalence in populations of injecting drug users JAMA. 1995 Oct 18;274(15):1226-31. 
18. Cárdenas S, Rivera N, Boteman JR, Aguirre P. Renacer una propuesta para volver a nacer. Fundación Renacer, UNICEF. Bogotá: Ágora; 2000.

19. Edinburgh L, Saewyc E, Thao T, Levitt C. Sexual exploitation of very young Hmong girls. J Adolesc Health. 2006 Jul;39(1):111-8.

20. Silverman JG, Decker MR, Gupta J, Maheshwari A, Willis BM, Raj A. HIV Prevalence and predictors of infection in sex-trafficked Nepalese girls and women. JAMA. 2007 Aug 1;298(5):536-42.

21. Menick DM, Ngoh F. Seroprevalence of HIV infection in sexually abused children. Med Trop (Mars). 2003;63(2):155-8.

22. República de Colombia-Ministerio de la Protección Social, Instituto Nacional de Salud. Sivigila: Boletín Epidemiológico Semanal. Semana Epidemiologica \#7 Fecha: Febrero 15 al 21 del 2004.

23. Cabral GA. Drugs of abuse, immune modulation, and AIDS. J Neuroimmune Pharmacol. 2006 Sep;1(3):280-95.

24. Friedman $\mathrm{H}$, Pross $\mathrm{S}$, Klein TW. Addictive drugs and their relationship with infectious diseases. FEMS Immunol Med Microbiol. 2006 Aug;47(3):330-42.

25. Profamilia. Encuesta Nacional de Demografía y Salud, Salud sexual y reproductiva en Colombia. Bogotá; 2000.

26. Organización Internacional del Trabajo (OIT). Programa internacional para la erradicación del trabajo infantil. [Internet] . Disponible en: http://www.ilo.org/public/spanish/ standards/ipec/publ/law/ilc/ilc86/com_chil/index.htm Consultado Septiembre del 2008.

27. Farley M, Kelly V. Prostitution: a critical review of the medical and social sciences literature, Women \& Criminal Justice. 2000; 11 (4): 29-64. 